\title{
VIPMOS, a Buried Local Injector for EPROMs
}

\author{
R.C.M. Wijburg, G.J. Hemink, L. Praamsma, J. Middelhoek \\ University of Twente, IC-Technology and Electronics department \\ P.O. box 217, 7500 AE Enschede (NL)
}

\begin{abstract}
A highly effective substrate hot electron injection EPROM device can be made using a buried injector, which operates in punch-through mode. The buried injector is formed by a local overlap of the $\mathrm{N}$-well and $\mathrm{P}$-well of a retrograde twin-well CMOS-process. As the VIPMOS-EPROM is compatible with VLSI-processing and the danger of latch-up doesn't exist, the VIPMOS-structure may be used in VLSI-applications. Due to an efficient electron supply mechanism as well as a high injection probability, programming rates of $1 \mathrm{~V} / \mu \mathrm{s}$ can be obtained.
\end{abstract}

\section{Introduction}

Substrate Hot Electron (SHE) injection is an attractive alternative to the conventional Channel Hot Electron ( $\mathrm{CHE}$ ) injection for programming EPROM's. SHE injection offers a higher injection efficiency and less stress on the gate oxide because of the distributed charge transport through the gate oxide (figure 1 \& 2). However, SHE injection has been unsatisfactory until now, because an efficient mechanism to supply electrons underneath the gate was not available [1], [2].

\section{Buried Injector}

A Vertical Injection Punch-through based MOS-structure (NIPMOS) can be used for a highly effective SHE injection EPROM (figure 3). The EPROM has an injector, which is formed by a local overlap of the $\mathrm{N}$-well and P-well of a retrograde twin-well CMOS-process. The VIPMOS-EPROM is compatible with this process. During programming, the injector is grounded and a high voltage (e.g. 15V) is applied

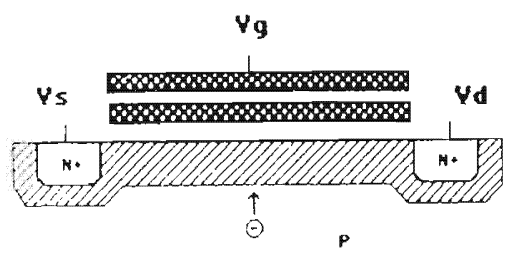

fig. 1: SHE injection



fig. 2: CHE injection 


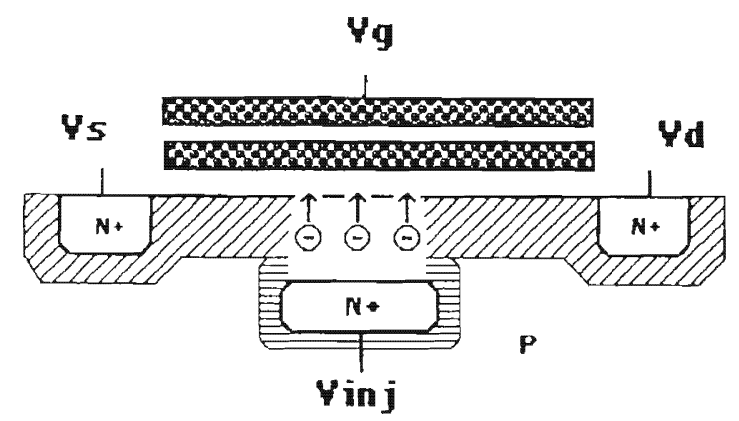

fig. 3: VIPMOS-EPROM with the buried local n-injector

to the control gate. Increasing the voltages of both source and drain will extend the depletion layer under the gate into the direction of the injector. At a certain voltage $\mathrm{Vpt}$, punch-through occurs and electrons are injected into the depletion layer under the gate. Some electrons become hot and will surmount the $\mathrm{Si} / \mathrm{SiO} 2$ potential barrier. Simulation results [3] show (figure 4), that the VIPMOS-structure very well supplies the electrons, which should be accelerated.

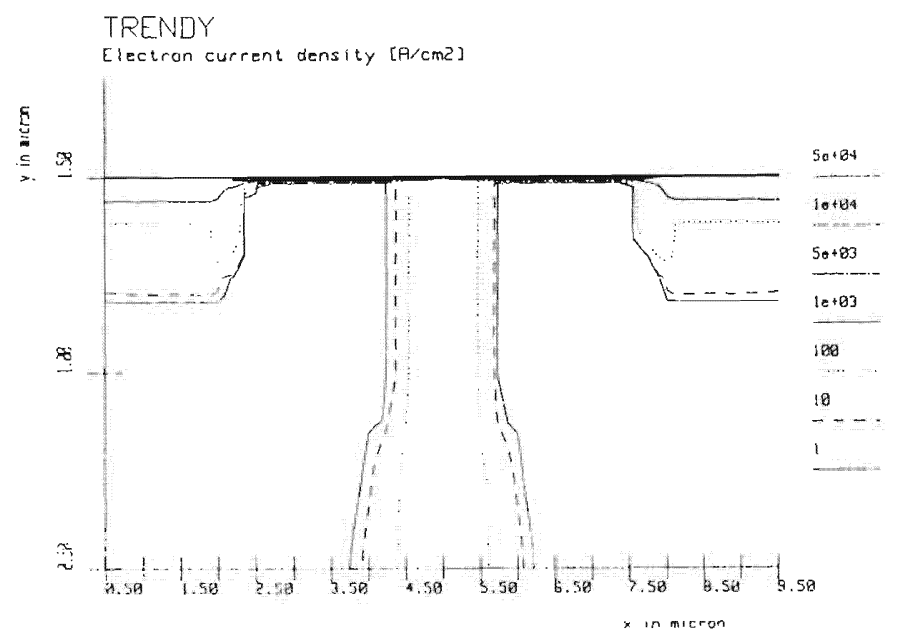

fig. 4: Simulated electron current density in programming mode $\mathrm{Vd}=\mathrm{Vs}=5 \mathrm{~V}, \mathrm{Vinj}=0, \mathrm{Vg}=10 \mathrm{~V}, \mathrm{Vb}=0 \mathrm{~V}$ )

The electron current density from injector towards the channel easily exceeds $100 \mathrm{~A} / \mathrm{cm}^{2}$. The injection only occurs in vertical direction, which makes this device suitable for VLSI-application without the danger of latch-up. As the potential of the floating gate decreases sufficiently, the punch-through condition cannot be satisfied any more and the injecting process automatically 
stops. During the read mode, the injector is connected to Vdd. Therefore, the potential barrier between the channel and the injector is raised and electrons are precluded from flowing into the direction of the gate.

\section{Measurements}

In figure 5a, the measured drain/source-current (Ids) and the "floating" gate current (lg) as a function of the drain/source to bulk voltage Nds) are shown. The voltage applied to the "floating" gate $\mathrm{Ng}$ ) is $12.5 \mathrm{~V}$. The source- and drain potential are equal. In figure $5 \mathrm{~b}$, Ids and $\mathrm{lg}$ as a function of $\mathrm{Vg}$, with $\mathrm{Vds}$ is $5.5 \mathrm{~V}$, are shown. The injection area of this device is $2.5 * 5.0 \mu \mathrm{m}^{2}$. The voltage, at which punch-through occurs, is about $4.8 \mathrm{~V}$. However, Vds has to be increased with a small amount to attract the electrons, which are not able to surmount the $\mathrm{Si} / \mathrm{SiO} 2$ barrier, from the channel.

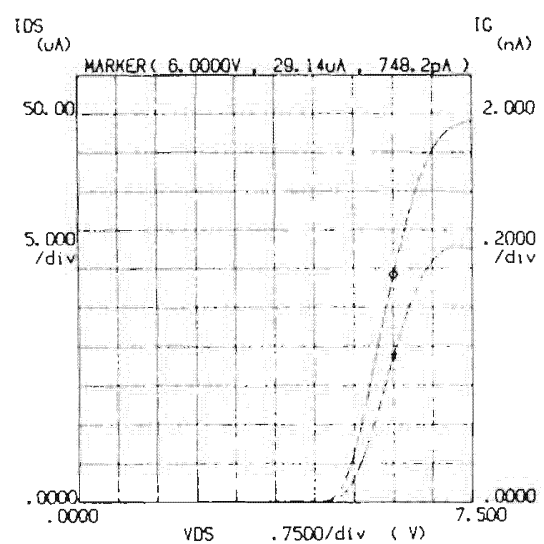

(a)

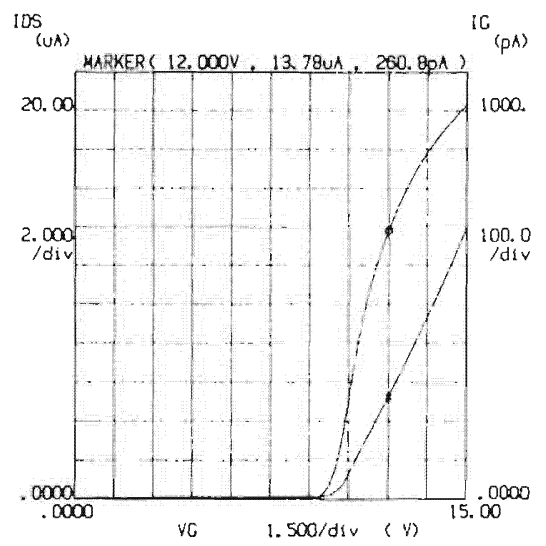

(b)

fig. 5: Drain/source and gate current as a function of $\mathrm{Vds}$ (a) and $\mathrm{Vg}$ (b)

By varying the doping-profile of the $P$-well, the punch-through voltage and thus the injection probability (Pinj $=\mid \mathrm{g} / \mathrm{Ids}$ ) can be varied. Figure 6 shows the measured injection probability at two different electrical-field strength's (Eox) in the oxide. The solid lines in the figure present the theoretical curves according to the lucky electron model [4]. The parameters are the same as used in [4], while the doping concentration has been fitted by a parameter extraction program [5].

Due to an efficient electron supply mechanism as well as a high injection probability, a high electron current density through the gate oxide can be obtained. We have measured current densities up to $1 \mathrm{~A} / \mathrm{cm}^{2}$ for the devices with a 
punch-through voltage of $8 \mathrm{~V}$. This implies, that depending on the ratio of the injection area versus the floating gate area, programming rates of $1 \mathrm{~V} / \mu \mathrm{s}$ can be obtained for the devices with a punch-through voltage of $8 \mathrm{~V}$. On the other hand, Vpt can be adjusted to a value of $4 \mathrm{~V}$. As a result, a $5 \mathrm{~V}$-only VIPMOS-EPROM should be possible.

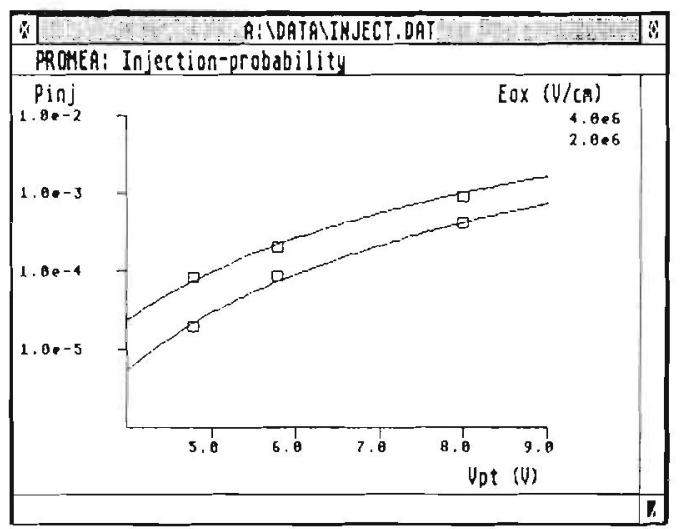

fig. 6: The measured and theoretical (solid lines) injection probability as function of the punch-through voltage

\section{Conclusion}

A local buried injector offers new possibilities for programming EPROM's using SHE injection. By means of punch-through, the local buried injector can effectively supply the electrons, which should be accelerated. Therefore, the injection of electrons doesn't occur into the lateral direction. This makes this device suitable for VLSI-applications without the danger of latch-up. The injector is formed by a local overlap of the $\mathrm{N}$-well and P-well of a retrograde twin-well CMOS process and is compatible with VLSI-processing. Oxide current densities of $1 \mathrm{~A} / \mathrm{cm}^{2}$ have been measured as a result of the effective injection mechanism and the high injection probability.

\section{Acknowledgements}

The authors would like to thank the Dutch Foundation for Research on Matter (FOM) for the financial support, G. Boom for his valuable discussions and support, L.F.P. Warmerdam and F.W. Ragay for their encouragements.

\section{References}

1. J.F. Verwey, J. Appl. Phys. 44, 2681 (1973)

2. B. Eitan et al., IEEE Trans. Elect. Dev. ED-31, 934 (1984)

3. E. van Schie et al., Nasecode VI conf. (1989)

4. T.H. Ning et al., J. Appl. Phys. 48, 286 (1977)

5. P.B.M. Wolbert et al., Nasecode VI conf. (1989) 